\title{
INTERACTIVE NATURAL IMAGE SEGMENTATION WITH REGRESSION BASED CLUSTERING ALGORITHM
}

\author{
David Solomon Raju Y ${ }^{1}$ and Dr. D. Krishna Reddy ${ }^{2}$
}

\begin{abstract}
The scope of this paper is to segment an object from the natural image irrespective of its background. This paper presents a novel approach of region based segmentation with linear regression employed with it. The proposed approach separates foreground and background pixel values based on an interactive labeling from the user. Interactive based segmentation is of great importance in research since it provides more accurate results than the learning based segmentation algorithms. The proposed is applied on several images from the available standard databases and also the performance is assessed with regards to several existing approaches. Results show that the proposed approach is consuming very low time for processing.
\end{abstract}

Keywords - region based segmentation, global colour distribution, active contour, edge based segmentation

\section{INTRODUCTION}

Image segmentation is considered as one of the main solutions to many computer vision applications and it is the process of dividing the image into distinct regions preserving its homogeneity. Interactive image segmentation involves minimal user interaction to extract the user's region of interest. It is of high interest area of research because it achieves satisfactory results than un-attainable automatic image segmentation algorithms. There are two main requirements for this interactive image segmentation process (i) the algorithm should be able to produce segmentation results that reflect user's intent (ii) the algorithm should be so efficient in providing the instant feedback. However, the intuitiveness of the user is allowed till the desirable results are attained.

Interactive image segmentation process can be categorized into two categories namely hard and soft segmentation algorithms. Firstly, the Hard segmentation algorithms as proposed in [1] [2] produces a binary map of the pixels which belongs to either fore-ground or back-ground and the later soft segmentation approach as stated in [3] [4] extracts a fractional part of an image. Interactive image-segmentation algorithms utilize either region properties like Adobe's magic wand or it may use boundary properties like active contour models [5]. In active contour methods, the segmentation results are obtained by placing a contour nearer to the boundary there by it could be able to grab out a certain boundary region. This method suffers from a major drawback is that the contour may be likely to be trapped in local minimum. On contrast, the intelligent scissor

\footnotetext{
${ }^{1}$ Department of Electronics and Communication Engineering HITS COE, Hyderabad, Telangana State, India

${ }^{2}$ Department of Electronics and Communication Engineering Professor, Chaitanya Bharathi Institute of Technology, Hyderabad, Telangana State, India
} 
algorithm expects the user to place points along the desired contour. The proposal mentioned in the paper is belongs to one such type of algorithm where the foreground and background pixels are marked with different colour tone by the user in an interactive manner.

The rest of the paper is organized as follows. section I explains about the need for intuitive segmentation and its application and also describes about different methods involved in it. Section II explains about the related work done so far in the field of interactive segmentation, section III explains about the proposed approach its importance and mathematical analysis. Section IV gives us results obtained from the proposed approach ending with conclusions.

\section{RELATED WORK}

Many researchers have focused on this interactive based image segmentation such as grab cut method as proposed in [6] [7]. In this analysis both regional and boundary properties are considered. With these methods an image is modeled as a graph. The node of each branch represents a pixel where the adjacent two neighboring nodes are connected with a weighted edge defined as the distance between the pixel values. The limitation of this grab cut method is that it considers an assumption that an object's shape is best described by its shape with smallest boundary length which cannot be true for very complicated shapes like hair and bushes more over these methods do not perform well in noisy environment.

Random walk based segmentation and its extensions as stated in [2][8] in which the image is modeled as a graph and found that it is better performing than the graph cut algorithm. Even this random walks segmentation requires the label elements representing the foreground and background region, however, these random walks lacks a global colour distribution which is very sensitive to the position and quantities of foreground and background seed points. In [9] Xiang et.al has spline regression based segmentation which is a combination of Green's function and Sobolev functional space. The main advantage of this approach is smooth and able to approximate the interpolation values at the scattered data points with arbitrarily controllable accuracy.

This paper presents a similar approach as stated in [9] but the difference in the clustering algorithm where the author in [9] uses K-means clustering for the partition of the labeled foreground and background pixels. This clustering approach sufferers from few demerits like uncertainty due to the presence of random initial seed points, this may be overcome by using fuzzy based clustering approaches. The most important property of this method is that the adjacent neighboring pixels are highly correlated. However, the very next pixels do possess nearly same feature data. This spatial relationship between the neighboring pixels constitutes a great aid in segmenting an object in image. Spatial clustering approach incorporates spatial information, however the objective function is derivative of weighting function which is altered after the clusters in neighborhood are considered.

\section{PROPOSED APPROACH}

Consider an image 'I' with ' $\mathrm{n}$ ' pixels to be segmented in which two sub sets foreground (F) and background (B) were present. Both these subsets are marked up by the user in an interactive manner. Assign a class label of ' +1 ' to fore ground and ' -1 ' to background points. Now the task is to partition the entire image pixels into these labeled partitions this can be achieved through a smoothing function

$$
\left.l(f)=\sum_{i=1}^{n f}\left(1-f\left(X_{i}^{F}\right)\right)^{2}+\sum_{i=1}^{n b}\left(1-f\left(X_{i}^{B}\right)\right)^{2}\right)
$$


Where $X_{i}^{F}$ and $X_{i}^{B}$ corresponds to number of foreground and background pixels

Where $f(x)$ can be represented as $f(x)=\alpha_{0}+\sum_{i=1}^{n} \alpha_{i} x_{i}+\sum_{j=1}^{n f} \varphi_{j}^{F}(x)+\sum_{j=1}^{n b} \varphi_{j}^{B}(x)$ here $\varphi_{j}^{F}(x)=\left|x-x_{j}^{F}\right|^{2} \log \left(\left|x-x^{F}\right|\right)$ and similarly for $\varphi_{j}^{B}(x)$. By solving the above equation and applying thresholding we can obtain the labels of foreground and background pixels.

$l_{i}= \begin{cases}\text { foreground } & \text { if } f(x) \geq 0 \\ \text { background } & \text { if } f(x)<0\end{cases}$

Now the clustering algorithm [10] [11] is applied for these user intuitive labeled elements by doing so the insignificant labeled elements can be removed.

\section{EXPERIMENTAL RESULTS}

The proposed algorithm is tested and evaluated with both real time and standard Berkeley segmentation image database [12] [13]. The results are tabulated below

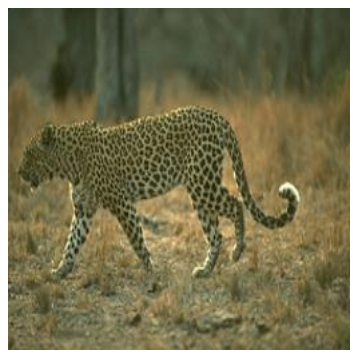

(a1)

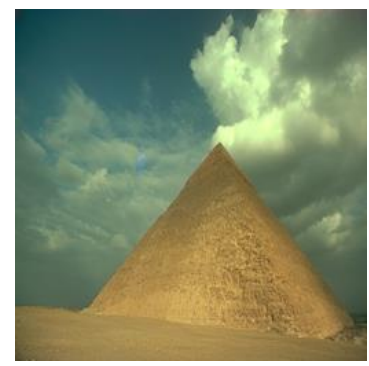

(a2)

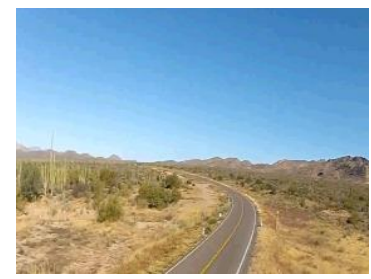

(a3)

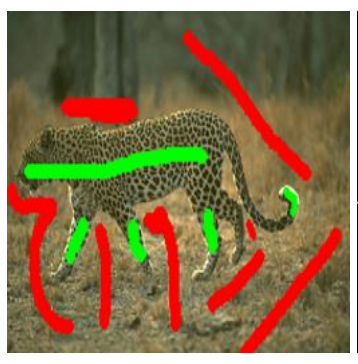

(b1)

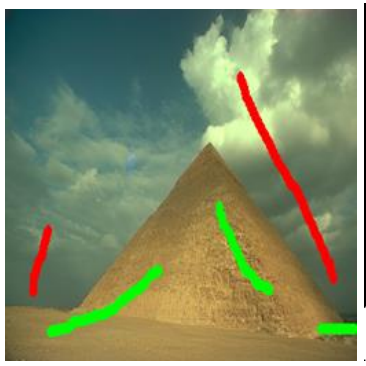

(b2)

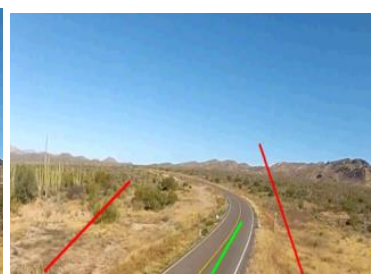

(b3)

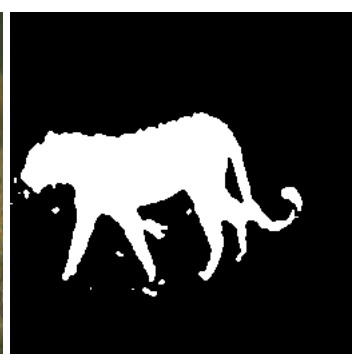

(c1)

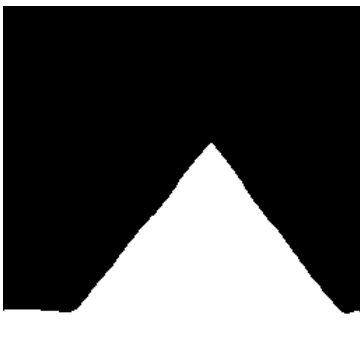

(c2)

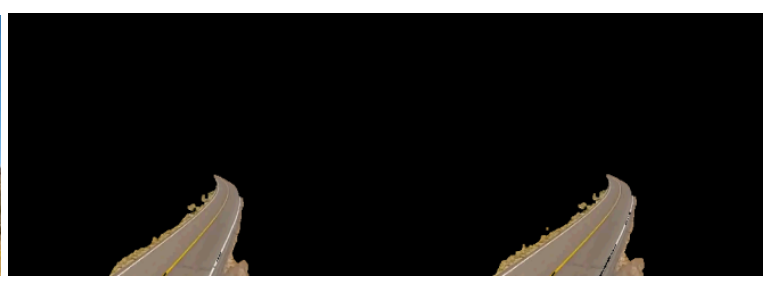

(c3)

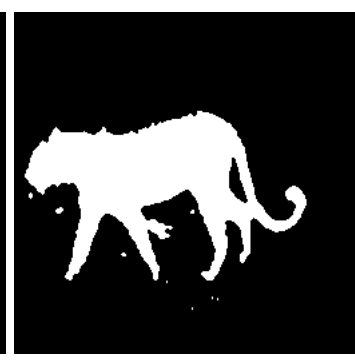

(d1)

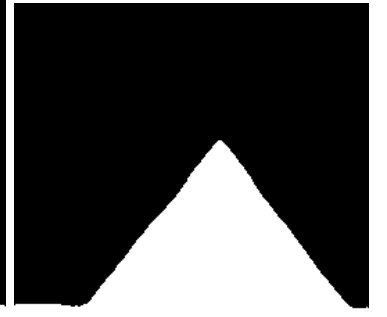

(d2)

(d3)

Figure 1: (a1-a4): original images (b1-b4): Labeled Images (c1-c4): segmented using regression based K-means (d1d4) Segmented using regression based SFCM

\section{CONCLUSION}

An interactive based image segmentation is proposed in this paper, the approach is modeled as a linear regression problem and also incorporating the concept of clustering analysis in order to obtain more efficient segmentation results. The approach is applied on both standard database and 
realistic image and results shows that it works perfectly to yield the most appropriate results when compared against ground truth images (Not displayed). This work can be further extended by incorporating edge based segmentation along with this region based approach.

\section{REFERENCES}

[1] Y. Boykov and M.-P, Jolly, "Interactive graph cuts for optimal boundary and region segmentation of objects in ND images," in ICCV, 2001

[2] L. Grady, "Random walks for image segmentation," IEEE Trans. PAMI, vol. 28, no. 11, Nov. 2006.

[3] A. Levin, D. Lischinski, and Y. Weiss, "A closed form solution to natural image matting," in CVPR, 2006

[4] A. Levin, A. Rav-Acha, and D. Lischinski, "Spectral matting," in CVPR, 2007

[5] M. Kass, A. Witkin, and D. Terzopoulos, "Snakes: Active contour models," IJCV, pp. 321-331, 1988.

[6] Y. Li, J. Sun, C.-K. Tang, and H.-Y. Shum, "Lazy snapping," in ACM Siggraph, 2004

[7] C. Rother, V. Kolmogorov, and A. Blake, "Grab Cut": Interactive foreground extraction using iterated graph cuts," in ACM Siggraph, 2004.

[8] L. Grady, "Multilabel random walker image segmentation using prior models," in CVPR, 2005.

[9] S. Xiang, F. Nie, C. Zhang and C. Zhang, "Interactive Natural Image Segmentation via Spline Regression," in IEEE Transactions on Image Processing, vol. 18, no. 7,2009.

[10] D. J. hemanth, D. Selvathi and J. Anitha, "Effective Fuzzy Clustering Algorithm for Abnormal MR Brain Image Segmentation," 2009 IEEE International Advance Computing Conference, Patiala, 2009

[11] D. Selvathi and R. Dhivya, "Segmentation of tissues in MR images using Modified Spatial Fuzzy C Means algorithm," 2013 International Conference on Signal Processing , Image Processing \& Pattern Recognition, Coimbatore, 2013

[12] https://www2.eecs.berkeley.edu/Research/Projects/CS/vision/bsds/

[13] http://host.robots.ox.ac.uk:8080/pascal/VOC/voc2007/

[14] K.-H. Tan and N. Ahuja, "Selecting objects with freehand sketches," in Proc. Int. Conf. Computer Vision, Vancouver, BC, Canada, 2001, vol-1

[15] A. Protiere and G. Sapiro, "Interactive image segmentation via adaptive weighted distances," IEEE Trans. Image Process., vol. 16, no. 4, Apr. 2008 